\title{
THE VIEWPOINT PARADIGM: A SEMIOTIC BASED APPROACH FOR THE INTELLIGIBILITY OF A COOPERATIVE DESIGNING PROCESS
}

\author{
Pierre-Jean Charrel \\ Université Toulouse 1 \& Institut de Recherche en Informatique de Toulouse, \\ 21 Allée de Brienne, F-31042 Toulouse Cedex, France. \\ tel: (33) 561128799 \\ fax: (33) 561128880 \\ Email charrel@univ-tlse1.fr, charrel@irit.fr
}

\begin{abstract}
The concept of viewpoint is studied in the field of the modelling and the knowledge management concerned in the upstream phases of a designing process. The concept is approached by semiotics, i.e. in dealing with the requirements so that an actor gives sense to an object. This gives means to transform the intuitive concepts of viewpoint and relation between viewpoints into the Viewpoint Paradigm: the sense of an object is the integration of the viewpoints which exert on it. The elements of this paradigm are integrated in a general model, which defines two concepts formally: Viewpoint and Correlation of viewpoints. The Viewpoint Paradigm is then implemented in operational concerns which are related with the intelligibility of the designing process. Two models of viewpoint and correlation are proposed. They raise of viewpoints management such as one can identify them in the written documents of a project.
\end{abstract}

\section{STUDY FRAMEWORK: THE VIEWPOINT CONCEPT}

\section{Viewpoints in everyday life}

The meaning most commonly given to the word viewpoint is that of a position providing a better view than other places. The summit of Mount Rhune, for instance, affords a magnificent view of the French Basque coastline from Hendaye all the way to Bayonne. The existence of a viewpoint implies that there is something to view, in this case the Basque coast, and someone to view it, i.e. one of the thousands of tourists who daily take the little rack-and-pinion train up to the viewpoint. Furthermore, the conditions of observation make the viewpoint more or less attractive - clear weather and a good pair of binoculars are, for example, ideal viewing conditions. Lastly, the viewpoint stops being a viewpoint when the object observed stops being observable, such as times when mist caps the Rhune, the binoculars break or snow prevents the little train from reaching the summit.

In the light of the above, the viewpoint is the meeting place of a given natural "object" (the Basque coast), a context (the weather conditions and observation devices available) and an actor (the tourist). The observation allows the actor to access to a representation of the object

A viewpoint has also the meaning of a specific opinion produced in someone by observing a specific object. In the world of film critics, for example, the book "Hitchcock" (Rohmer E., Chabrol C. (1988)) contains a transcription of the viewpoints of Eric Rohmer and Claude Chabrol towards the film Rear Window by their fellow director Alfred Hitchcock. Although the film still constitutes an object, it is, this time, an artificial one. The representation of the object, i.e. its screening at a matinee, is also given. Moreover, the viewpoint is shared by two actors: their opinion is presented, and represented, in a book as a joint opinion. What we have here is a transcription of one of the two film directors' opinions, but one they did not want to personify. Also, this opinion only exists because other opinions exist. Finally, criticism can be seen as the representation of another kind of film on a different plane to that of the screen: a reader of the book can thus form his/her own opinion of the film by reading Eric Rohmer and Claude Chabrol's opinion, even if they have never seen the film.

This two examples bind the notion of viewpoint exerted on an a given object, to the notions of actor, representation, and context. In the first case, the representation is also given, in the second case, the actor who exert his viewpoint produces a new representation of the object, accessible to other actors.

Let us now consider a situation where the object is not given, but produced. It is encountered in the theatre. By definition, a play is the meeting point of the two kinds of viewpoints discussed above, and two production processes.

We are looking at three objects here: the written work, the play and the performance. The play as an object only exists if several viewpoints - the director's and the actors' - meet and correlate at each rehearsal throughout the production process. The play is the product of a process at the end of which these viewpoints finally converge. The end of the production process coincides with the beginning of the performance organisation process. Each performance has its own context: place, date and time. Other viewpoints now come into play: those of each spectator. The spectator, in a sense, is also an actor participating in the performance through his attention and reactions. Moreover, each performance contributes to the play and affects the subsequent performance. An exemplary case is Jérôme Deschamps' play "Lapin chasseur". The stage and theatre are divided into two parts, one representing a restaurant kitchen, the other the dining hall. From their seats, the spectators can only see one of the two areas although they can hear stage noise and laughter from the spectators on the other side. During the 
interval, the spectators change sides and the actors perform the play again: from the viewpoint of the spectators on one side of the stage, the actors and spectators on the other side are part of the play.

In this example, a viewpoint is attached to a given object - the script, the play, or a performance -, to a actor - a comedian, the director, or a spectator - and to a context - the rehearsal hall, the date, the place and time of a performance. And two additional elements now appear:

- a produced object: the play, the performance

- a collective production process of this object.

The object produced changes depending on the viewpoint. .

Thus, from the director's viewpoint, the play is his representation of the script and is produced from the actual written work. The play is also the last rehearsal, at the end of the production process.

From an actor's viewpoint, the play is also a representation of the written work at the end of the production process and becomes an object on the basis of which a "representation" is produced in the context of the organisation process.

From a spectator's viewpoint, a performance "represents" the play, but it is also a given object. Actually, the performance is a "representation" of itself, the polysemy of the term "representation" (i.e. as a synonym of "performance") standing as proof of this reflexive relationship.

\section{Viewpoints for designing technological systems}

The theatre metaphor introduces the objects that will be discussed in this paper, i.e.: the technological systems, and more particularly space systems and software systems.

Let us consider the early designing phases, i.e. the phases during which requirements are analysed and specified. During these phases, the object being designed exists only in the form of a project. It is only a reality in the minds of the client, the contractor, the members of the project team and future users. In other words, the object only exists from the viewpoint of the actors involved. To design the object, these actors will be called upon to come up with a series of intermediate, transitory and accessory objects as well as specifications, prototypes, test sets, beta versions, etc., which can be seen as traces or representations of the different viewpoints exerting an influence on the future object. These representations are expressed during the validation phases of the project when different viewpoints converge, confront each other and correlate. The result of the final validation is the object itself.

We can therefore say that the viewpoints on the object precede the object. This is reminiscent of the theatre play in its rehearsal phases, the play being the epitome of an artificial object if ever there was one, and thus a good metaphor of a technological system.

\section{An object as an integration of viewpoints?}

Let us pursue the discussion on viewpoints in each of the previous situations. Ultimately, every object is relative to a viewpoint: if an object can be observed or referred to using a word, then someone must exist to observe or refer to it. Hence, at least one viewpoint on the object must exist. Likewise, we can only describe the objects in the previous situations from the viewpoint corresponding to our position: as far as the Basque coastline is concerned, the tourist's viewpoint is not the geographer's nor the guide book writer's. All these actors have their "own" Basque coastlines. The viewpoints of both directors towards the film by their famous colleague are not identical and are not the same as those of a professional critic, the film's producer or a simple spectator, although in "Rear Window" Hitchcock places the spectator in the director's shoes. And a play is not the same seen from the stage, backstage or the theatre above all if the theatre is split into two sections.

The following question naturally arises as a consequence:

Can an object be the integration of all the viewpoints exerted on it?

For the Basque coastline, this would consists in defining it as the amalgam of all the elements used to write a guide book, all elements relating to its geography and all other considerations relative to the Basque coast. This is tantamount to reducing the object to the aggregate of its viewpoints. However, no matter how numerous they may be, are the representations of the Basque coast ever one with the actual coast?

It is not, in fact, the entire Basque coast that is invoked, but its visible portion only and the person(s) seeing it. "Rear Window" takes on a sense when we attend a screening of the film, read a critical article, or give our own opinion on it; the main concern of the director, actors and spectators of the play "Lapin chasseur" is each individual performance; once launched into space, the spacecraft is only accessible via its representation, i.e. the signals it exchanges with the ground sector. Lastly, the software system is an abstract object and therefore its own representation, given to its users.

The artificial objects we are concerned with here, the play, the spacecraft and the software system cannot be reduced to the aggregate of their representations, but they could not be produced without the existence of at least one viewpoint. 
It is the question of the object status to be produced with respect to its representations which is now posed. We do not intend to take up a stance among the formidable philosophical arguments raging on the conditions governing the existence or reality of an object or an idea. Rather, our objective is to highlight the value of the viewpoint concept in attempting to explore this issue.

The few examples above are intuitive illustrations of the viewpoint concept and its corollaries in everyday situations, art, science and technology where the concept becomes meaningful. They will serve as a reference to investigate the question posed.

\section{How this paper is organised}

This paper summarises studies and experiments on the viewpoint concept that we performed during the knowledge modelling and management phases early in the designing process of a new product, spacecraft or software system.

The next section begins to reconcile the viewpoint concept with the precepts of semiotics on the conditions necessary for a actor to give an object sense. This reconciliation provides the wherewithal to transform our intuitive notions on viewpoints into a paradigm. The elements of this paradigm are then integrated into a general model which formally defines two concepts: Viewpoint and Correlation of Viewpoints. Lastly, we will present a translation of this model into an object formalism.

Then we present an implementation of the viewpoint paradigm. It gives rise to operational concerns relating to the intelligibility and control of the designing process, particularly controlling project convergence towards a final specification. This section presents two models of viewpoints and correlations: they issue from an experiment performed as part of a research effort (Galarreta D., Charrel P.J., Rothenburger B \& al. (1998)Charrel P.J., Rothenburger B., Trousse B. \& Vogel C. (1998)) aimed at recognising and eliciting viewpoints and their correlation on the basis of written documents produced during a collective designing activity. The models are presented in an object-oriented formalism.

At least we summarise related works investigating the viewpoint concept in the fields of requirement engineering, and concluding remarks about this initial anchor point for the viewpoint paradigm.

\section{VIEWPOINTS AS A PARADIGM FOR DESIGNING}

In the introduction, we described situations suggesting that viewpoints could be the key of a paradigm in the sense given by Thomas Kuhn and whose characteristics are defined by Edgar Morin in (Morin E. (1991)): as a social issue, a paradigm is a vision of the world that is neither verifiable nor disprovable, which is accepted as an axiom, excludes the issues it does not recognise, generates a feeling of reality and is recursively connected to the reasoning and systems it generates.

The statement on which our paradigm is founded could be the following:

The sense of an object is the integration of the viewpoints directed at it.

Two key points give rise to a definition of the elements in this paradigm: the viewpoint concept is central to two processes, the process whereby an object achieves sense and the process whereby actors in the project aimed at designing this object communicate amongst each other.

The first key point is to take into consideration only objects that have a sense and actors that give these objects sense. In this way, the object to be designed and an actor participating in the project are not isolated entities: the object has sense when it is connected to how it is interpreted by an actor in a context through a representation that takes on the form of a statement using a symbology. Any representation of an object is thus subjective and contextual.

This identifies in a wider sense the object's sense and the result of the process used to design this object.

The second point arises out of the following observation: the act of designing a space system, a satellite and its ground segment for instance, brings into play a great many technical, organisational and financial skills to find solutions to problems such as: electrical power supply, technical constraints, signal transmission, controlling cost prices, and managing and coordinating teams, over a period that may last for a very long time. It can be said that all actors contributing one of these skills to the project has his or her "own" satellite that must be integrated with that of his partners.

The quality of communications between project participants is therefore a key factor of the project's success. Indeed, in this concurrent engineering context, the actors are exchanging partial, incomplete and even contradictory representations. The basic idea of the research we undertook was to no longer only take into account the representations of the future object, but also the object itself and its designing process in all their manifold complexity, in the sense given by J.-L. Lemoigne to this term as per his definition of a complex system (Lemoigne J.L. (1990)): "... a system that cannot be reduced to a finite model, no matter how complicated, stochastic and sophisticated it may be, and irrespective of its size, number of components and the intensity of their interactions". 
This position is conducive to a global - systemic - view of objects, actors, representations and the design process.

Semiotics is encountered at this point in its two flavors, English and French. We now demonstrate that the viewpoint concept is based on the concept of "sign". We shall then go on to define the elements of our viewpoint paradigm. Lastly, we present a model of these elements in an object-oriented formalism.

\section{The semiotic foundations of the viewpoint concept}

Semiotics postulates that the only sources of sense are differences and provides a set of tools to identify them. Today, semiotics covers a number of "sign" theories: the sign is considered by some to be an element of the meaning process and as an element of the communication process by others. In each of these approaches, we will recognise several elements connected to the viewpoint concept discussed in the first section.

\section{VIEWPOINTS AND MEANING PROCESS}

Peirce's English language semiotics defines a sign as "something that takes the place of something for someone with some respect for some reason" (Peirce C.S. (1932)). According to Umberto Eco (Eco U. (1980)) "With some respect" means "that the sign does not represent the entirety of the object but rather - through various abstractions - represents it from a certain viewpoint or with a view to a certain practical use". Claude Vogel adds in (Galarreta D., Charrel P.J., Rothenburger B \& al. (1998)) that "Peirce's sign is conceptual and relative" and quotes Peirce: "nothing is s sign if it is not interpreted as such". Thus, according to Peirce, a sign only acquires the status of a representation of an object in a relationship of three terms encompassing the object, the sign as a signifier or expression, and the signified as the content of the expression.

The triad (object, signifier, signified) is often represented in the "semiotic triangle". Figure 1 shows this triangle and gives some of the equivalent terms used by Saussure, Morris, and Hjelmslev (Eco U. (1979)).

Peirce adds two notions to his semiotic triangle: the first is that a content can itself be an expression (a sign), and the second is that the context decides which content to associate with the expression to give it sense. Claude Vogel quotes Pierce (Galarreta D., Charrel P.J., Rothenburger B \& al. (1998)): "Suppose we hear a word out of context, the word "Grenade" for instance. (This) word has a sense. I know this for sure but I do not know which sense. I will only know if the sign "Grenade" points to another sign acting as its interpretant, "city", "fruit", or "weapon". Let admit... I come from Spain. The word "city" is not... sufficient to me. But the interpretants of "Grenade" do not stop there, in the syntactic order: Spain, Iberian Peninsula, Europe, Mediterranean sea, Arab occupation, Islam, architecture, Generalife, Alhambra, garden, lion, an so on".

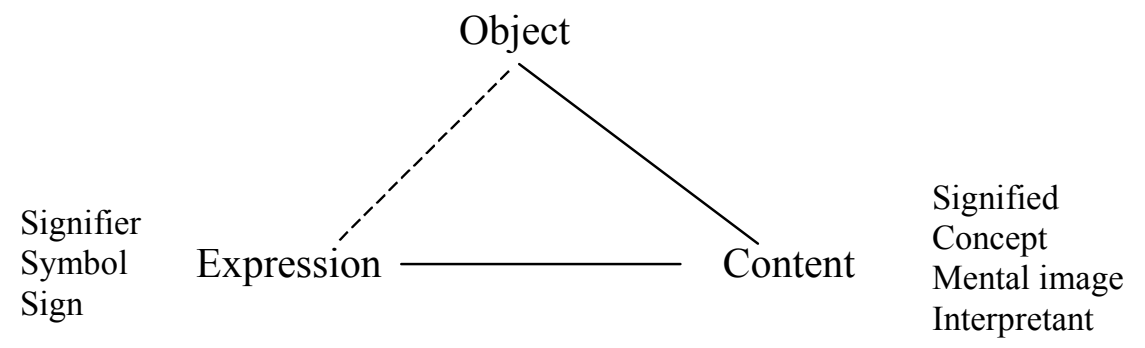

Fig. 1 The semiotic triangle

In the archetype of the meaning process represented by the semiotic triangle we find several elements connected with our viewpoint notion: the object and the conditions for an expression to be qualified for representing an object.

\section{VIEWPOINTS AND COMMUNICATION PROCESS}

In our work on multiple viewpoint design, the links between the actors in the design project were also taken into consideration so that the representations they produce have a sense, i.e. so that they can be understood, evaluated, revised, modified and assembled with a view to final consistency.

In the case of a space system, the first steps in the designing process generally involve producing documents in natural language. "Formal" specification documents are only produced at the end of the process. All these documents are therefore expressions of viewpoints and their links.

We now encounter the second semiotic foundation of the viewpoint in connection, this time, with the French language approach.

According to this approach (Deledalle G. (1990), Greimas A.J. (1979)), semiotics provides a tool for visiting a document like a monument. It thus presents text as being inseparable from the author and the reader: the sense of a text is that given to it by its author, which is also the sense retrieved by the reader. Thus we may observe that a 
text is a communication medium if a reader is able to retrieve a sense from it, albeit a different one. A text thus only contains conditions placed by the author for retrieving the sense, these conditions being relative to the form - structure, presentation -, the literary genre, the language - English, terminology -, the style, etc. A coauthored text is already the product of a pooling of several sense retrieval conditions, i.e. those recognised by its authors.

According to the philosopher Bernard Stiegler 1 "All fabricated objects constitute memory because they are traces of an action". This states the fact that a fabricated object constitutes the trace of an action whose results are stored in the form of an object. In this respect, a text is an incomplete object: if, in the first degree, it is the memory of an act of writing, it requires another action for a sense to be reconstructed from it. A text is never completely finished: the reader always adds the follow-on. In other words, a text is a kind of specification that must be realised by the reader. It is a class whose instances are the multiple interpretations of its readers.

The French semiotic method is conducive to highlighting the differences in a text. These differences are the sources of sense. They are elicited using three types of analysis. The first analysis consists in identifying "narrative programs" or contracts: these feature an "actant" - person or thing - that performs or has an action performed which is then evaluated. Its opposite is an anti-narrative program which creates an opposition and is a source of sense. The second analysis looks at how the roles and interaction of the actants combine. Roles are expressed on the basis of canonical types. The third analysis identifies the "isotopies" of the text, i.e. its themes and the related elements of the text.

All these constructions are representations of the text. They throw direct light on invisible aspects of it. These are, in fact, relationships that can be presented in the form of graphs showing terms and concepts that although present in the text, cannot be isolated from it because of the latter's linear form.

Other elements linked to the viewpoint concept are thus found in these French semiotic analysis schemas: actors, objects, and relations between actors and objects.

\section{Remark}

The absence of the interpret in Peirce's semiotic triangle does not exclude the communicational aspect of the sign. Peirce also formed the semiotic triangle for communication-related purposes: "the interpretant is the idea the sign gives birth to in the mind of the interpret - even if the presence of the effective interpret is not required" (Eco U. (1979)). According to Jürgen Habermas's commentary (Habermas J. (1991)), "before accomplishing its function as a representation, the sign must also be interpretable". Peirce excludes the actor and the context from the semiotic triangle in order not to attach the object's signifier and the signified to them, but he explains that "something cannot stand for something else without standing for someone".

\section{Elements of the viewpoints paradigm}

Reconciling the viewpoint notion with semiotics first leads to a formal definition of the basic concept of Viewpoint and then to a set based representation which is used to define this of Viewpoint Correlation Let us assume a given universe of discourse.

\section{Definition 1}

A Viewpoint is the interpretation of an Object by an Actor: It is defined by the Object on which the interpretation is performed, the Actor performing it, the Expression and Content of the interpretation of the Object by the Actor, and the Context in which this interpretation is performed.

A Viewpoint thus comprises five poles:

- The Actor holds at least one Viewpoint, in the Context of which he or she produces an interpretation of the Object.

- The Object is the integration of the interpretations produced by the actors exerting a Viewpoint on it.

- The Context is the condition governing the way the Actor exerts his or her Viewpoint. It can signify, according to the case, either the place from which the Viewpoint is exerted, the moment in time it is exerted, the tool used by the Actor to exert his or her Viewpoint.

1 Conference given by Bernard Stiegler on 3 February 1996 in Blagnac during the "From the body to the technological corpus (du corps au corpus technologique)" talks. 
- The Expression is a statement, expressed in a symbol system, that is attached to the Object by the Actor within the Context of the Viewpoint to express his or her interpretation of the Object.

- The Content is the meaning given within the Context by the Actor to the Object by means of Expression.

The semiotic triangle does not mention the Context in which the sign is produced nor the Actor that produces it. A Viewpoint can be considered to be a semiotic triangle situated for its Actor in his interpretation Context.

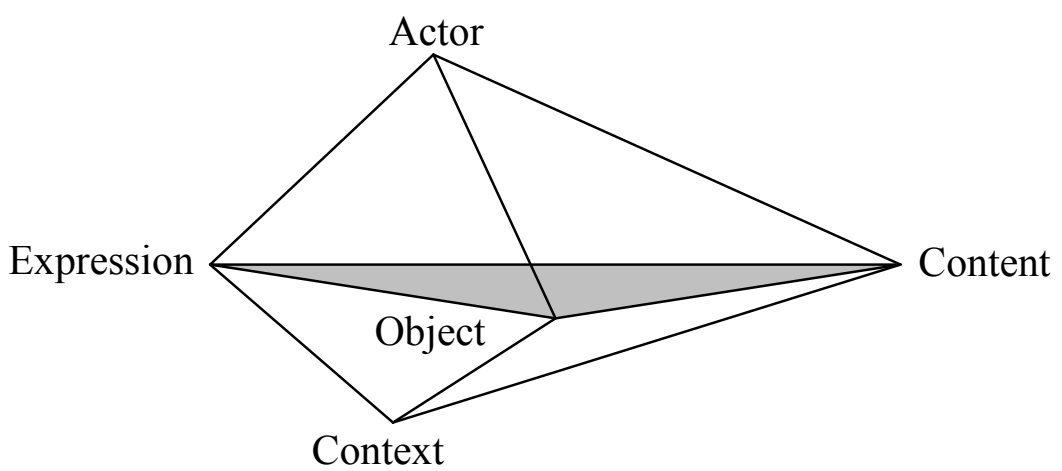

Fig. 2 Viewpoint and semiotic triangle

Figure 2 proposes a representation of the Viewpoint as a quintuplet of poles:

$<$ Actor, Object, Context, Expression, Content $>$

In our paradigm, we do not attempt to define the Object and the Actor before the Viewpoint. The Viewpoint is pre-existent to the Object and the Actor. Here, the concept of Viewpoint is given a status of paradigm in the sense of Emmanuel Levinas who founds his paradigmatic method on the idea that "...it is the act that makes the form emerge in which it recognises its model, unseen until this instant " (Levinas E. (1994)).

The Content, certainly the most "abstract" of the five poles, contributes information, or rather knowledge, given by the Viewpoint on the Object. In this connection, let us quote G.G. Granger who wrote in (Granger G.G. (1994) p. 33) "... to say that a notion has a content is presupposing that by attributing it to a subject or by entering into a relationship, it provides us with information. Information is expressed in a statement virtually opposed to many other statements which, from a certain viewpoint, can be seen to have the same form".

When the symbolic system used in Expression is a formal one, i.e. when semantics is associated with each statement, the two poles Expression and Content merge and correspond to what we invoked at an earlier stage by the term Representation.

\section{Definition 2}

The Representation of a Viewpoint is the pair $<$ Expression, Content $>$.

\section{Definition 3}

The View of a Viewpoint is the triplet $<$ Expression, Content, Context $>$

\section{Definition 4}

The Universe $\mathrm{P}$ of viewpoints is the Cartesian product:

$$
\mathrm{P}=\mathrm{A} \times \mathrm{O} \times \mathrm{C} \times \mathrm{E} \times \mathrm{CO}
$$

in which $\mathrm{A}, \mathrm{O}, \mathrm{C}, \mathrm{E}, \mathrm{CO}$ designate respectively the aggregate of Actors, Objects, Contexts, Expressions, and COntents in the universe of discourse.

We use dotted notation to designate one of the components of a viewpoint. For example, p.a designates Actor a of Viewpoint $\mathrm{p}$ in universe $\mathrm{P}$.

For the following definitions, the Universe is implicit. Actually, it is the reference from which all the viewpoints can be defined. 


\section{Definition 5}

We will use $\langle X>$-correlation to designate any transitive relationship on $\mathrm{X} \times \mathrm{X}$, where $\mathrm{X}$ designates one of the five sets of poles or the Cartesian product of several of them.

\section{Examples}

- $\mathrm{An}<\mathrm{A}>$-correlation is a transitive relation on $\mathrm{A} \times \mathrm{A}$.

- $\mathrm{A}<\mathrm{C}, \mathrm{E}>$-correlation is a transitive relation on $(\mathrm{C} \times \mathrm{E}) \times(\mathrm{C} \times \mathrm{E})$.

This notion is extended to viewpoints in the following way:

\section{Definition 6}

Two Viewpoints $\mathrm{p}$ and $\mathrm{p}^{\prime}$ are said to be $<\mathrm{X}>$-correlated if an $<\mathrm{X}>$-correlation exists between two of their poles.

\section{Examples}

- In the universe of discourse of an organisation, the relationship "works in the same department" is an $<\mathrm{A}>$-correlation.

- In situations where the object is given, whether natural or otherwise, viewpoints are naturally correlated by the Object, or $<\mathrm{O}>$-correlated, and the relationship is the identity. This is the case, for instance, of the Basque coast, where the $<\mathrm{O}>$-correlation is the identity relationship.

- For the example of the film "Rear window", three $<\mathrm{O}>$-correlated viewpoints exist: the viewpoints exerted by each of the two directors, and the viewpoint whose Actor is the pair (Chabrol, Rohmer), whose Expression is the critic on "Rear Window", and whose Context is the book "Hitchcock".

\section{Definition 7}

When two Viewpoints are correlated by Expression and Content, i.e. $<$ E, $\mathrm{CO}>$-correlated, they are said to be correlated by the Representation, or $<\mathrm{R}>$-correlated.

\section{Definition 8}

An $<\mathrm{X}>$-Correlator is a function:

cr : $\mathrm{X} \rightarrow \mathrm{X}$

where $\mathrm{X}$ is one of the sets of poles or a Cartesian product of several of them.

\section{Notations}

When there is no ambiguity, cr is said to be a Correlator. 2 viewpoints $\mathrm{p}$ and $\mathrm{p}^{\prime}$ correlated by cr are crcorrelated.

\section{Remark}

The transitive closure of a correlator defines a correlation.

This notion is extended to viewpoints in the same way as $<\mathrm{X}>$-correlation.

\section{Examples}

- In the universe of an organisation, "is the hierarchical superior of " is an $<\mathrm{A}>$-correlator over all viewpoints.

- Suppose that the Expressions of two viewpoints $\mathrm{p}$ and $\mathrm{p}^{\prime}$ are two knowledge bases made up of logical formulae, an $<\mathrm{E}>$-correlator can be constructed to define the formulae of $\mathrm{p}^{\prime}$ deducible from $\mathrm{p}$.

\section{Definition 9}

A system of viewpoints $\mathrm{S}$ is defined by the $\mathrm{n}$-uplet: where:

$$
<\mathrm{A}, \mathrm{O}, \mathrm{C}, \mathrm{E}, \mathrm{CO}, \mathrm{P},<\mathrm{X}>\text {-Correlations, }<\mathrm{X}>\text {-Correlators }>
$$

- $\quad$ A, O, C, E, CO designate sets of Actors, Objects, Contexts, Expressions and Contents,

- $\mathrm{P}$ designates the universe of viewpoints, 
- $\quad<\mathrm{X}>$-Correlations and $<\mathrm{X}>$-Correlators designate the two sets of Correlations and Correlators defined on $\mathrm{P}$

\section{Definition 10}

Let $\mathrm{S}$ be a system of viewpoints. A set SP of viewpoints of $\mathrm{P}$ is said to be correlated if there exists a correlator cr of all X-Correlators of S such that the viewpoints of SP are correlated 2 to 2 by cr.

\section{Definition 11}

Let $\mathrm{S}$ be a system of viewpoints. A graph GP of viewpoints on S is defined as follows:

- a node of GP is a viewpoint of P,

- $\quad$ an edge between two nodes $\mathrm{p}$ and $\mathrm{p}^{\prime}$ exists if there exists a correlator $\mathrm{cr}$ of $<\mathrm{X}>$-Correlators such that $\mathrm{p}$ and $\mathrm{p}^{\prime}$ are cr-correlated.

\section{Graph of viewpoints and intelligibility of a designing process}

According to the viewpoint paradigm, the process whereby a technical object is collectively designed gives rise to a particular viewpoint graph.

Here, the contexts of all viewpoints are the various milestones of the process.

There exists an initial and a final node respectively related to two viewpoints whose actor is the project's customer, and intermediate nodes which are the various viewpoints exerted throughout the process.

For the initial viewpoint, the Object is the assignment on the customer's purchase order, the Context is the instant the project is launched, and the Representation is the entire set of documents produced for the customer and the design team.

For the final viewpoint, the Object is the designed product - and all the knowledge acquired on its manufacture, maintenance and operation -, the Expression is the integration of all the Expressions of the viewpoints in the final Context, and the Content then represents the formal acceptance by the customer of the designed Object.

The product only exists physically at the end of the designing process, and it is the Object of all the viewpoints exerted in the final Context of the process. The process is complete when the viewpoint of the customer Actor is able to prevail.

Let us consider the graph of viewpoints for a designing process. We classify the correlators into two types: Static correlators and Dynamic correlators.

Dynamic correlators are relative to the design process and support the intelligibility of the process: they ensure its visibility and coherence as discussed in (Charrel P.J., Galarreta D., Hanachi C., \& Rothenburger B. (1993), Charrel P.J., Rothenburger B., Trousse B. \& Vogel C. (1997)). The Context pole suits to represent the time scale of the process. So, dynamic correlators are $<\mathrm{C}>$-correlators, where $\mathrm{C}$ Context is Time.

Static correlators are all $<\mathrm{X}>$-correlators defined at the other poles or combinations of $<\mathrm{C}>$-correlated viewpoint poles. They can take on the form of reasoning on viewpoints to organise, where necessary, their consistency. Reasoning on viewpoints and the process facilitates the discovery and management of all significant differences. Here, we once again encounter the semiotic rationale for viewpoints: when an Actor produces a new Representation during the design process, differences arise along the Actor and Object axes Actant $^{2}$ and Time in semiotic axiology.

\section{Examples}

Two remarkable situations can arise in a design process, which are interpreted using Static and Dynamic correlators:

- "Validate a Representation" is an $<\mathrm{R}>$-correlator which links the Viewpoint $\mathrm{p} 1$ of the Actor who produces the Representation to be validated and the Viewpoint $\mathrm{p} 2$ of the actor who validate it. The $<\mathrm{E}>$ component is the identity relationship, and the $<\mathrm{CO}>$-component is the boolean function: $\mathrm{p} 1 . \mathrm{CO} \rightarrow$ true, false \}.

- Objects are created throughout the process. They are assembled and modified to constitute the final body of specifications. Let us consider the history of the different versions of an Object produced by the same Actor during the process. Each version is related to one Viewpoint, and all these Viewpoints are

\footnotetext{
${ }^{2}$ Unlike French semiology, a distinction is made between objects and actors
} 
linked to each other by $<\mathrm{A}, \mathrm{O}, \mathrm{C}>$-correlators where the $<\mathrm{A}>$ and $<\mathrm{O}>$ component are the identity relationship, and the $<\mathrm{C}>$ component is the relationship "next step".

\section{Representation model for viewpoints and correlations}

Object-oriented formalisms are, actually, candidates for serving as a formal media for representing viewpoints and correlations between viewpoints. Figure 3 presents a model of a graph of viewpoints, inspired by the metamodel of the UML language classes (Rumbaugh J., Jacobson I. \& Booch G. (1998)).

The overall composition of a viewpoint is indicated in this model as a class related to five other classes that correspond respectively to the Actors, Objects, Contexts, Expressions and Contents of the Universe of viewpoint. The class Poles aggregates the five associated classes with the Viewpoint class. The cardinalities 0,1 express the primacy of the viewpoint with respect to its poles. The cardinality * indicates that the components may appear in several viewpoints. A Representation necessarily comprises an Expression, with or without a Content, and a view is compulsorily composed of a Representation, with or without a Context.

The correlations are instances of a class of associations that link viewpoints two by two, and the correlators are the methods of this class. The correlators are declared as methods of the Correlation class.

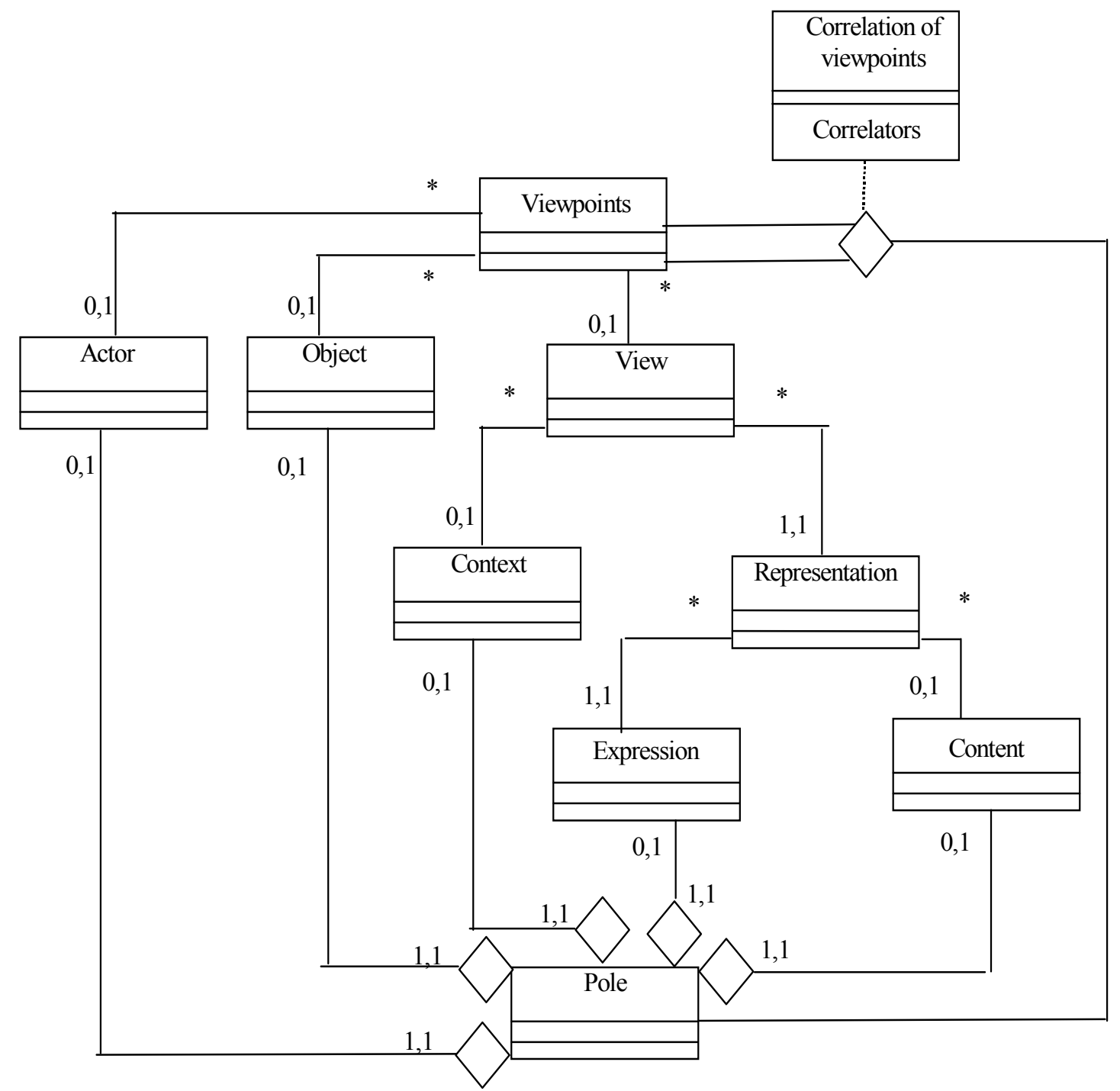

Fig. 3 Object-oriented model of a graph of viewpoints

\section{IMPLEMENTING THE PARADIGM OF VIEWPOINTS FOR THE MANAGEMENT OF A DESIGNING PROJECT}

The viewpoint paradigm generates specific representation models, two of them are presented in this section, for managing viewpoints generated by a designing project. In this section, we discuss propositions for viewpoint 
models inspired by the studies carried out as part of the research effort on dynamic viewpoints quoted in the first section (Charrel P.J., Rothenburger B., Trousse B. \& Vogel C. (1998)).

The overall objective of the research was to demonstrate by experiment that the notions of viewpoint and correlation could be used and useful to structure, understand and process the results of a content analysis of a discourse. Here, the discourse is composed of text that is rarely subjected to this type of analysis: a transcript of a conversation between three space technology engineers.

The global results are presented in the form of :

- a method and software tool for analysing texts used to identify viewpoints and their changes in the course of time - i.e. their $<\mathrm{C}>$-Correlation - throughout the project process,

- tool specifications to manage a viewpoints base supporting project management.

The framework of research was to study viewpoints and their correlation in the situations of a project for designing the architecture for a new little sized spacecraft. The objective was to provide tools to elicit the viewpoints brought into play during the design process and to highlight those which have effectively contributed to the success of the project. Feedback from the experiment enabled to specify a viewpoint-oriented prototype supporting design project management.

Initially, we set forth this project in the viewpoint paradigm and then briefly describe the analyses performed. Lastly, we specify two viewpoint management models. An object-based formalism is used for these models.

\section{The designing project in the viewpoint paradigm}

The context of the action is the following: a proposition for a specification was drawn up during a meeting between a project manager, named D1, and two engineers, named D2 and D3, in the space industry. A video recording and the written transcription of the discussions held during the meeting constitute the traces of the design process.

The conversation transcript is the trace of the interventions of the Actors D1, D2 and D3, but also of other Actors mentioned by D1, D2 and D3 - individuals, companies -, of Objects produced by the latter - "antenna", "propulsion system" -, and of their interactions during the design process with respect to the components of the Object being designed - layout of the satellite's propellant tanks, size of the antennae, mass of the components, etc.

This transcription, throughout the entire design process, of aspects relative to the structure, the functions, and the interactions between the different components of the spacecraft being designed can be interpreted as the modelling process of this object and its operating environment.

The analyst of the situation is himself or herself an Actor who exerts a Viewpoint the, Object of which is the designing process, i.e. the graph of converging viewpoints, the Context is the period of time the meeting lasted between the initial instant at which the Object was non-existent and the final instant at which the conversation was stopped by the project manager D1, and the Representation is the transcription of the discussion - video representations are not dealt with. This Representation is also that of the viewpoints exerted by the three Actors in the meeting on the Object to be designed. The Representations of these viewpoints are the interventions transcripts - by the Actors during the meeting, and their Contexts are the instants at which these interventions took place. The Object is constructed as dialog progresses by adding and reviewing partial Expressions in the viewpoints brought into play.

\section{Analysis of the experiment}

Let us sum up two analysis that Bernard Rothenburger and Claude Vogel respectively carried out (Charrel P.J., Rothenburger B., Trousse B. \& Vogel C. (1997)) on the discussion transcript.

First, the entire text was processed by a terminology classification software - Alceste ${ }^{\mathrm{TM}}$. This software used statistics to identify the major thematic classes represented in a text. Four classes were produced here. One of these classes includes quantitative notions: numerical values, units of measurement, comparisons between values, etc. It corresponds to the technical aspect of the discussion.

The text body was then converted into a table of pronounced words classified by speaker and by minute, which enabled to calculate usage frequency of the various classes per minute of statement of each actor, to make statistical estimates on the results, and to produce curves showing trends in these statistics.

The curve in Figure 4 shows that the designers D2 and D3 display a difference in how they deal with technical terms. Actually, D1 is the manager of the design group to which D2 and D3 belong. D1 starts off by explaining the issue by evoking its technical and quantitative components. At a later stage, D2 and D3, who have specific technical skills, appropriate these themes and look for solutions to the various problems posed by D1. The interpretation of the three curves are the following: between D2 and D3, the difference in how they deal with technical terms remains stationed in a fairly narrow interval while the difference they each display with respect to D1 increases regularly in time. 


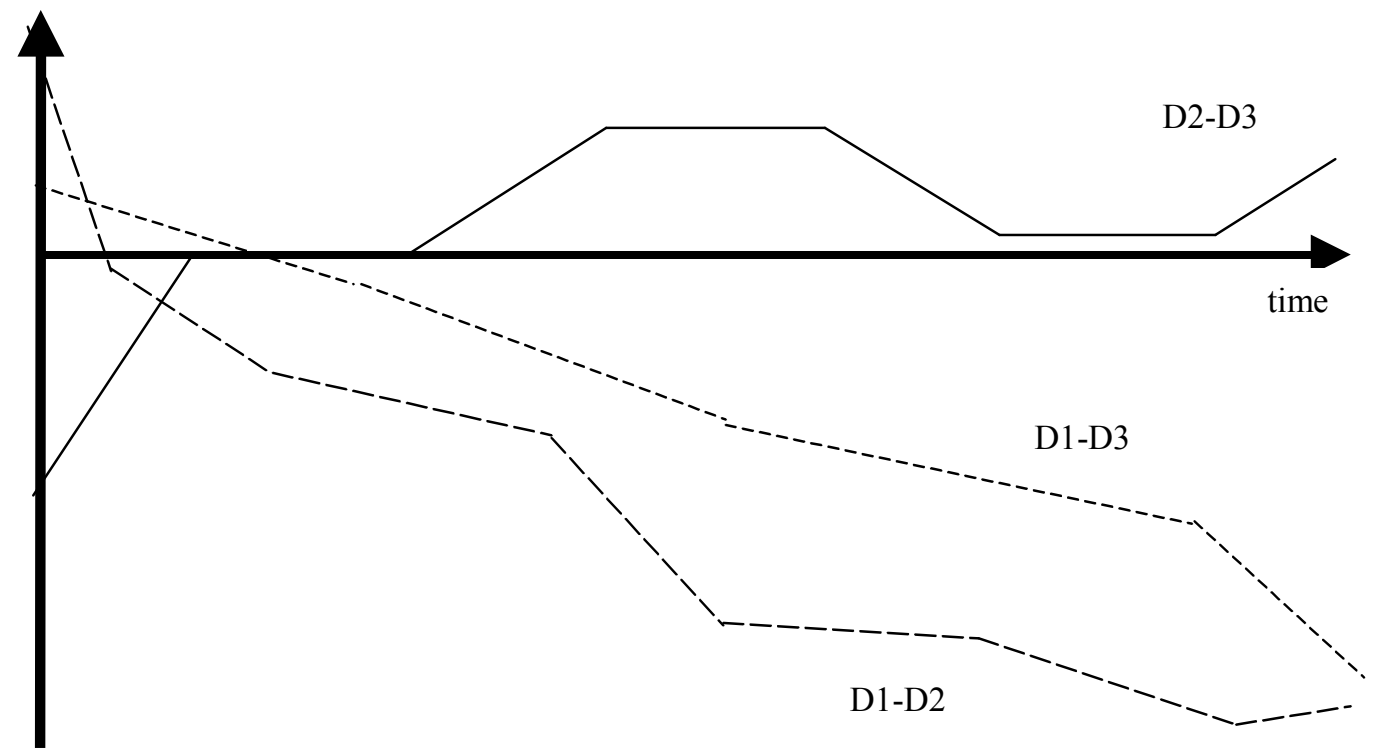

Fig. $4<A$, Infometrical $>$-correlation between viewpoints on technical terms

The second analysis used a statistical representation of the text as the source and not the text itself by means of SEMIOLEX ${ }^{\mathrm{TM}}$ tool. The text was divided into 6 periods of 30'. A glossary of keywords was selected from the general index of keywords constructed from the transcript. The keywords in the glossary occur at least twice in the index. On the basis of this glossary, the statistical analysis combines the two data representation planes built from the various samples: frequency distribution tables and co-occurrence tables.

Part of the results of statistical analysis is represented graphically by lexical networks attached to the cooccurrences. Figure 5 illustrates an example of a network and the excerpts from the relevant text: the term "shielding" appears as a pivot for neighbour terms such as "impact" or "pressure" and so defines a kind of semiotic proximity between them.

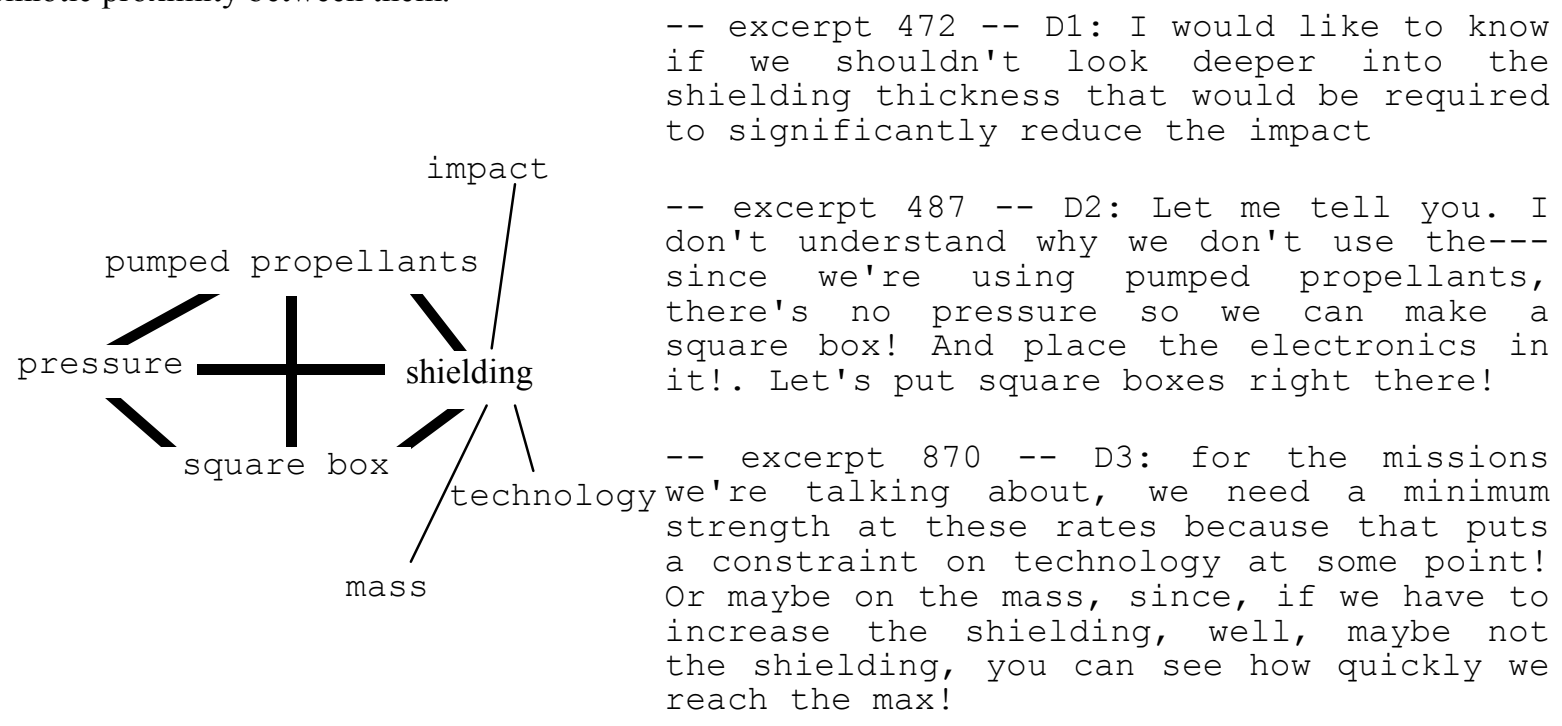

Fig. 5 Text excerpts with the lexical network which represent them

\section{Correlation models for the management of a designing project}

The experiments involving the acquisition and recognition of viewpoints from text performed during the abovementioned research work leads to a proposition for a tool to facilitate the project steering by its terminological and infometrical components.

The experiments have brought to the fore some remarkable forms or "regularities". For example, regularities occur during a particular period of the spacecraft design process. For example, the homomorphism of certain curves representative of the discussions between D2 and D3 involving design, or, on the contrary, the exactly opposite gradients of other curves as shown by other curves. 
We can now deduce the features of a viewpoint management tool capable of assisting actors exerting viewpoints by facilitating or enriching the analyses that have been conducted.

Schematically, our proposition consists in using an object formalism to represent items of information - partial, not consistent from one to another at any instant of the process, and revisable - about the actors participating in design, and their productions at the various stages when they are made visible by these actors.

Object-based approaches are an answer to controlling the design process because they provide systematic means for translating the technical, functional and usage components of the spacecraft. For example, the spacecraft's specification can be translated by a class diagram, its operating specification by a use-case model and statetransition diagram.

We can reconcile viewpoints management operations and their corresponding actions expressed in their object representations.

\section{TERMINOLOGICAL CORRELATION}

Terminology is defined as a network of links between terms and concepts, where a term is composed of several words and designates a determined concept of a particular domain, and a concept is a piece of knowledge characterised by an informational definition and its position inside a network of concepts. In this network, two concepts are linked by relationships such as "is synonym of", "is a ", "is part of".

The viewpoint notion leads to stress, in a given designing project, several terminologies:

- viewpoint terminologies, each of them is local to a Viewpoint, and is part of its Context;

- the project terminology, where synonyms, common terms, shared concepts, are defined.

The project terminology is a $<\mathrm{C}>$-correlation between all the viewpoints, and is named Terminological Correlation. It takes into account the insertion/deletion of terms or concepts.

A query language and a visualisation tool can implement the Terminological Correlation. The query language can retrieve terms and concepts and their respective relationships, by means of operators like the followings:

- for a term: number of links with concepts,

- for a concept: integration/isolation - number of adjacent concepts - and density - number of linked concepts,

- for a network of concepts: dimension - number of nodes and edges -, and density -maximal distance between two nodes, ratio number of concepts/number of terms, ratio number of concepts/number of links.

The visualisation tool aims at presenting evolution curves of theses measures along the time.

Figure 6 presents a class schema which suits to the terminological correlation of the viewpoints. The corpus of written documents, from which terms are issued, are accessible via the "source documents" method of class Term. The "classification" method represents the access to classification tools like used in the quoted research project.

\section{INFOMETRICAL CORRELATION}

The reasoning is now based on the particular Representation of the text given by the lexical networks issued from the statistics processed by the statistical tool used in the quoted research action.

The infometrical aspect of the viewpoints enriches the analysis, by offering selected views of the corpus owing to the networks of co-occurrences. Among these views are:

- the network local to a viewpoint, by selecting terms which co-occur with the Actor of the Viewpoint,

- the network limited to a given instant of the designing project,

- the networks the form of which is remarkable.

Like terminological networks, there are several possible measures, accessible by a query language or a visualisation tool:

- the number of links between a term and other terms or other networks,

- the integration or isolation of a network - number of adjacent network -, the density of a network number of linked terms, number of links in the network,

- the dimension of the network constituted by all the networks - number of nodes, number of links -, the network density - maximal distance between two nodes, ratio number of networks / number of terms, ratio number of networks / number of links. 


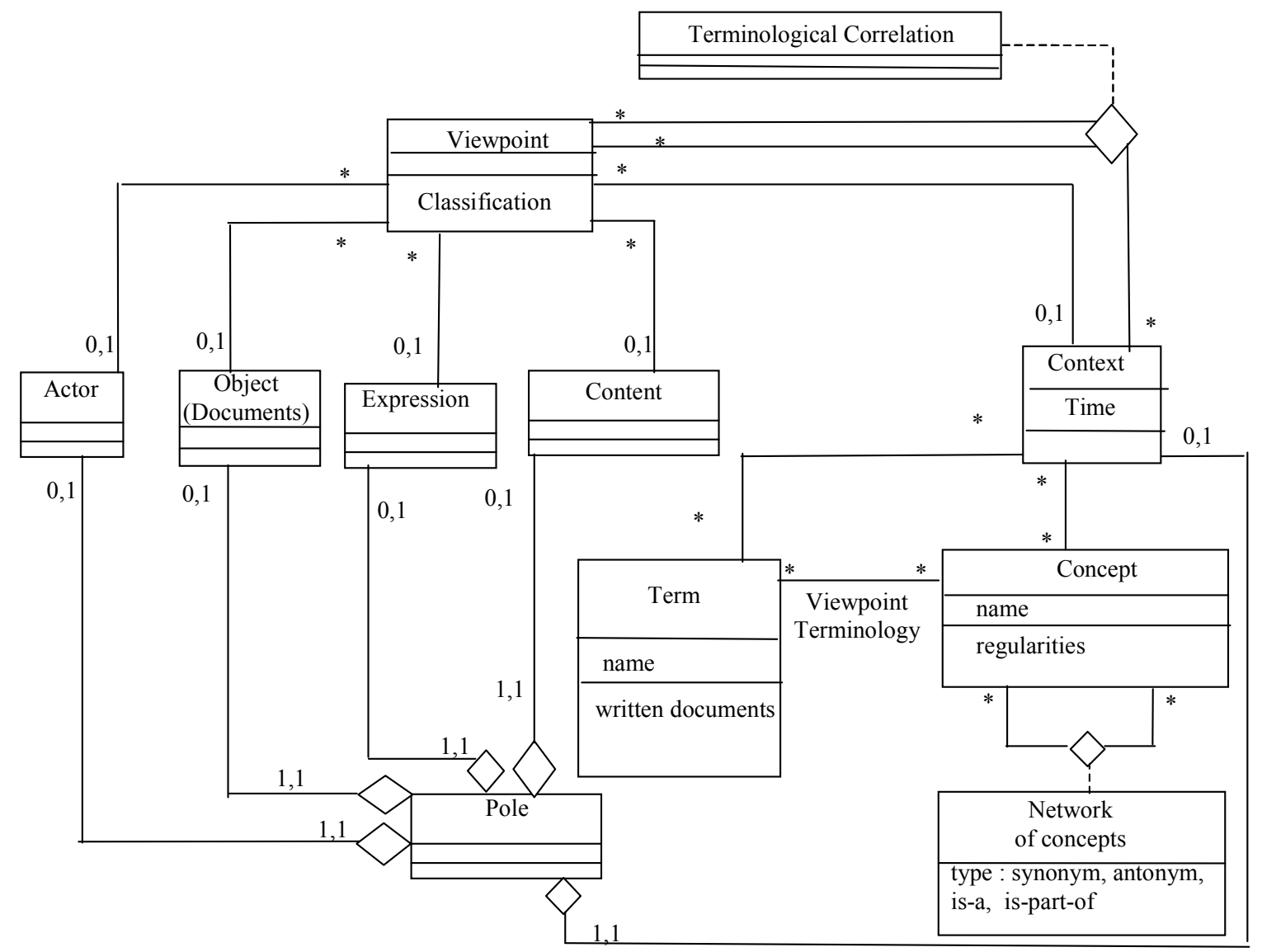

Fig. 6 Object-oriented model of a Terminological Correlation

For corpus of texts where chronology is a significant parameter - as the documents of a project - a computeraided management tool of the history of the Representations of the Viewpoints can provide qualitative measures like:

- the trajectory of a term in the different viewpoints, i.e. its appearance and disappearance, increase of cooccurrences with other terms or progressive isolation,

- scenarios issued from an infometrical analysis, i.e. recognition of regularities in the networks with reference to types of scenarios, acquisition of new scenarios.

Figure 7 presents a class schema which suits to the infometrical correlation of the viewpoints.

These correlations can carry out two types of control actions. The first action relates to the management of the evolution of the lexical networks. Each Actor adds or modifies documents related to a particular feature of the project, or constitute the basis of a new viewpoint. Then, these documents will add new terms and new links to the networks. The management of these extensions consists of measuring their influence on the quoted measure indexes. The second action aims at reducing the distance between two networks. Synonymy and contradiction between two terms present in two different viewpoints can leads to likenesses in the networks. Looking for these likenesses may, on the other hand, lead to collect synonyms and contradictions. The actors of the project are then able to simulate the actions to execute in order to delete these contradictions, or take these synonyms into account. 


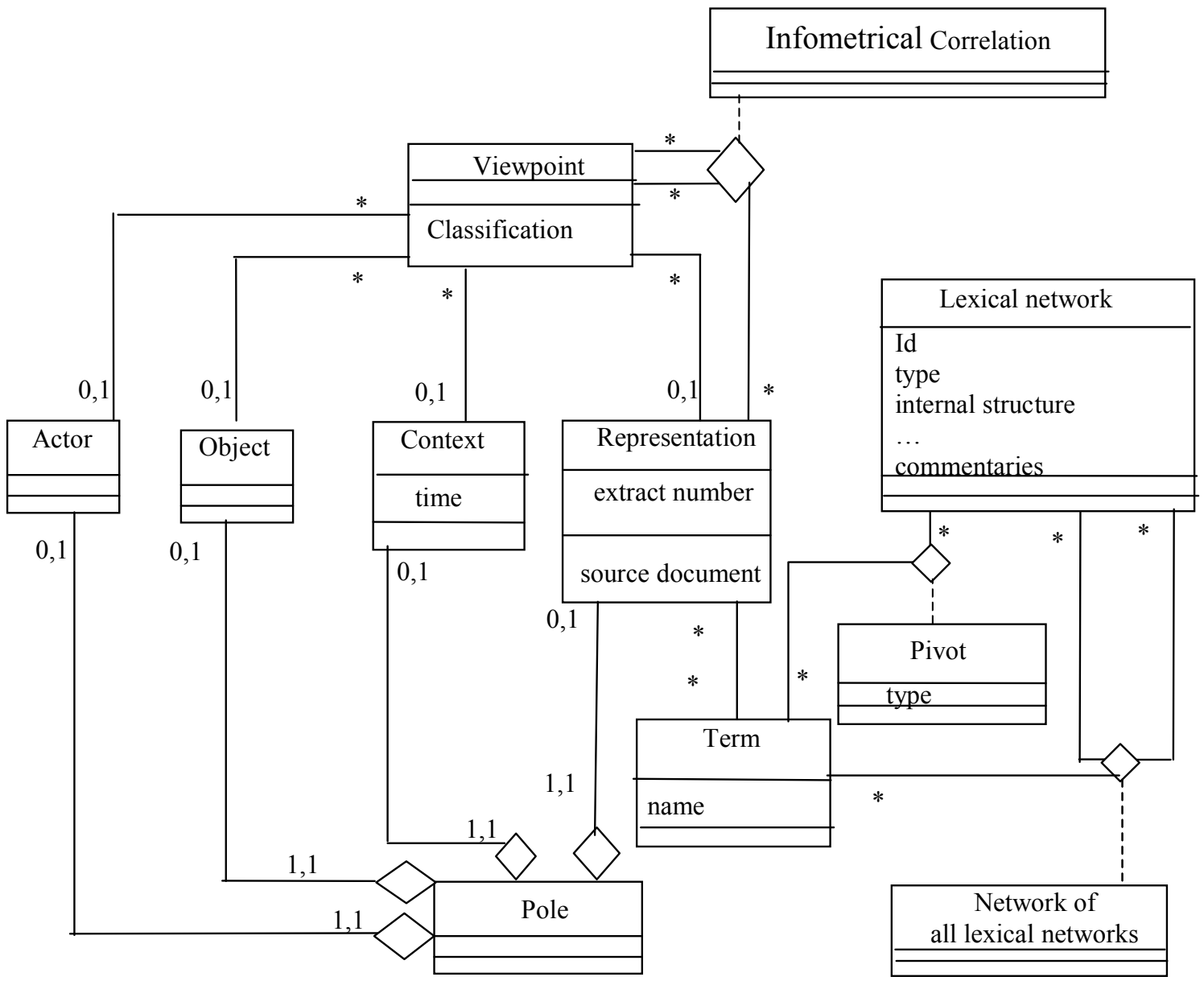

Fig. 7 Object-oriented model of a Infometrical Correlation

\section{RELATED WORKS AND CONCLUDING REMARKS: VIEWPOINTS IN REQUIREMENT ENGINEERING}

The requirement engineering activity is known to collect knowledge, methods, techniques, tools able to analyse and specify all functions and properties that a system to be designed must hold to achieve its mission (Nuseibeh B., Kramer J. \& Finkelstein A. (1994)) .This activity is also described as the production process of functional and non functional requirements (Leite J.C.S.P. \& Freeman P.A. (1991)).

P. Zave mentions in ((Zave P. (1997)) about fifteen problems to be solved which relates to the requirement engineering activity. She identifies three classes of problems: (1) objectives, functions, and constraints investigation of the future system, (2) behaviour specification of the future system, (3) evolution management of the future system.

Requirement engineering is basically a multi-disciplinary operation, as states P. Zave, which put together many actors, each of them holding his or her own perspective on the system (Easterbrook S.M., Finkelstein A., Kramer J. \& Nuseibeh B. (1994)). So, the definition of the requirements of a complex system put into play multiple viewpoints using various skills, responsibilities, and knowledge.

The concept of viewpoint has already been instantiated in requirement engineering. J.C.S.P. Leite defines a viewpoint in (Leite J.C.S.P. (1988) ) as an attitude or a mental position which a person adopts to observe the universe of the discourse. G. Kotonya and I. Sommerville refer in (Kontonya G. \& Sommerville I. (1992)) to an external entity which interact with the system being analysed. With this respect, their VOA project proposes to collect and solve requirements, classify and analyse interactions between viewpoints, and integrate functional and non functional aspects of the requirements. A. Finkelstein and H. Fuks speak in (Finkelstein A. \& Fuks H. 1989)) of an agent, logical participant to the dialogue who is responsible of the maintenance of a particular perspective. They define a viewpoint as an "object, weakly coupled, locally managed, encapsulating knowledge about the representation modes, the design process and partial specifications of a system and its domain". Formally, a viewpoint is defined as a tuple <style, plan, domain, specification, history $>$ where style is the used representation formalism, plan describe the actions, process and development strategies, domain describe the scope of the viewpoint towards the global system, specification describe the viewpoint domain in its style, and history keeps the trace of the actions executed during the development process. 
Two facets of the notion of viewpoint have been studied: the expression of requirements and the means to deal with them, and the organisation of the requirement acquisition process.

The description of a viewpoint poses two problems, due to the diversity: this of the formalism of the Expression pole, and this of the Content pole.

The diversity of the Expressions is inherent to the notion of viewpoint, since they must reflect the way the different Actors express. Nevertheless, using the same formalism facilitates the confrontation of the viewpoints during the validation phases of the project. So, until now, the heterogeneity of the formalism has never gone beyond the heterogeneity encountered in classical analysis methods: standard formalisms issued from Software Engineering - Data Flows, Entity-Relationships diagrams, Class diagrams, etc.

The diversity of the Contents originates from the fact that, at the beginning of the designing, each Actor holds his or her own concerns. We encounter once again the difficulty to put into relation formal specifications of distinct, or even independent, elements of the system.

As the designing process aims at building a collection of formal, rigorous and consistent specifications of the Object, some authors are looking for means to verify and/or enforce this consistency. This question is relevant to conflict resolution and refers to the cooperation and negotiation modes between agents. Requirement engineering is here defined as the process which identifies the differences between viewpoints, classifies these differences, evaluates several solutions and at last integrate viewpoints in a unique representation. J.C.S.P. Leite and P.A. Freeman's early validation relies on VWPL language (Kontonya G. \&Sommerville I. (1992)), which is the unique description language of the requirements. A comparison tool provides all the syntactic and semantic differences between the definitions. M. Ainsworth, S. Riddle, and P.Wallis aim at checking formally that any given set of viewpoints is valid (Ainsworth M., Riddle S., Wallis P. (1996)). They use Z specification of viewpoints to build an amalgamation of individual viewpoints into a single coherent whole. The amalgamation process is defined on a particular relation between specifications that is a conditioned refinement relation. $\mathrm{H}$. Bowman present in (Bowman H., Derrick J., Linington P., Steen M. (1996)) a framework for checking the consistency of the five viewpoints defined by the Open Distributed Processing standardisation initiative (ITU Recommendation X.901-904 - ISO 10746 1-4 (1995)). They define consistency as a relation between two specifications: two specifications are consistent iff a third specification can be found, which is a development (refinement or conformance)) of both specifications and the third specification can be implemented. A. Finkelstein and B. Nuseibeh accept temporary inconsistencies. They claim temporary inconsistencies are not only possible, but useful. They implement inconsistency by means of the following particular mathematical relation $\mathrm{R}$ between viewpoints: for each $\mathrm{p}$, there exist $\mathrm{p}$ ' such that $\mathrm{pRp}$ '. The source viewpoint $\mathrm{p}$ is responsible to verify the relation $\mathrm{R}$ with the target viewpoint $\mathrm{p}$ '.

About the relationships between viewpoints, B. Nuseibeh, J. Kramer and A. Finkelstein stress three questions in (Nuseibeh B., Kramer J. \& Finkelstein A. (1994)): (1) how to ensure their definition, (2) who takes them into account and (3) how to apply them. Actually, their proposition, based on CORE method (Mullery G.P. (1979)), defines viewpoint templates and a plan which comprises four actions: an action to create viewpoints, an internal consistency control action inside each viewpoint, a consistency control between viewpoints, and action triggers. These actions are executed according to the rules of a development model.

So, we share with almost all these authors, the opinion that the diversity of requirements is an unavoidable feature of a technological system, a software in particular. Two statements distinguish the quoted works from our proposition. At first, requirement engineering is generally considered as the earliest stage of a design process. We try to act still earlier, i. e. on specifications expressed by natural language, before their formalisation. The viewpoint paradigm dissociates Expression and Content, and thus allows to consider expressions which aare not provided with formal semantics, as those of the natural language. The second statement is concerned with the relationships between viewpoints. In many works, the objective is to ensure above all the formal consistency between viewpoints. We consider consistency is one possible $<\mathrm{E}>$-correlation, but is far to be the only relevant one. Actually, the two correlation models we propose in this paper can take consistency checking as a part., because consistency is one of the parameters of the convergence state of a designing process.

This paper put a first milestone of a theoretic and experimental foundation of the Viewpoint Paradigm. The presented models have not yet been validated by experiments in industrial large scale projects. This will be the next step of our work. The question of the computerised support of these object-based models must be studied in an existing environment, in order to integrate it as an easy desktop tool. These tools are also possible links with existing Technical Data Management Systems which are often based on object-oriented representation. Figure 8 shows a draft view of the architecture of a viewpoint manager system. 


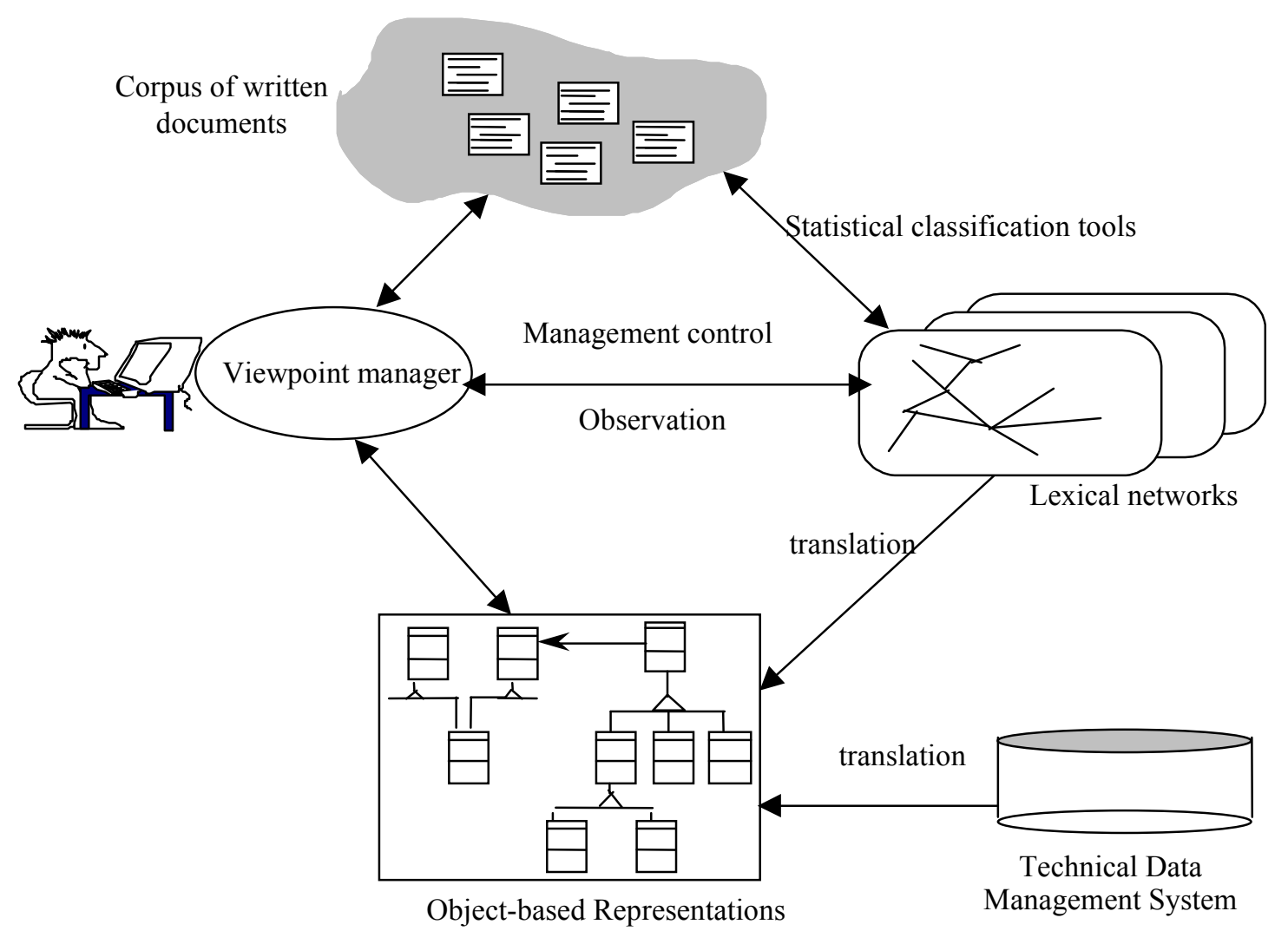

Fig. 8 Architecture of a viewpoint manager software

\section{REFERENCES}

Ainsworth M., Riddle S., Wallis P. (1996) "Formal validation of viewpoint specifications", Software Engineering Journal, January 1996, pp. 58-66.

Bowman H., Derrick J., Linington P., Steen M., (1996) "Cross-viewpoint consistency in Open Distributed Processing", Software Engineering Journal, January 1996, pp. 58-66.

Charrel P.J., Galarreta D., Hanachi C., \& Rothenburger B. (1993) "Multiple Viewpoints for the Development of Complex Software", Proceedings of IEEE Conference on Systems, Man and Cybernetics, Le Touquet, France, October 17-20, pp. 556-561.

Charrel P.J., Rothenburger B., Trousse B. \& Vogel C. (1996) "Etat de l'art sur les points de vue en Sémiotique, Linguistique et Informatique", Research report D1.1 of the project Etude des points de vue dynamiques, contract CNES/INRIA 871/94/CNES/1492.

Charrel P.J., Rothenburger B., Trousse B. \& Vogel C. (1997) "Modèles de corrélation de points de vue", Research report $\mathrm{D3}$ of the project Etude des points de vue dynamiques, contract CNES/INRIA 871/94/CNES/1492.

Charrel P.J., Rothenburger B., Trousse B. \& Vogel C. (1998) "Spécification d'une maquette démonstrative et application à l'exploitation d'informations du secteur spatial", Research report D4-D5 of the project Etude des points de vue dynamiques, contract CNES/INRIA 871/94/CNES/1492.

Deledalle G. (1978) "Commentaire", Ecrits sur le signe, C. S. Peirce, Editions du Seuil, Paris.. pp. $203-251$.

Deledalle G. (1990) Lire Peirce aujourd'hui, Giovannangeli D., Ed,. Le point philosophique, De Boeck. Brussels.

Easterbrook S.M., Finkelstein A., Kramer J. \& Nuseibeh B. (1994) "Co-ordinating Distributed Viewpoints: the anatomy of consistency check", Proceedings of Concurrent Engineering Research and Applications, West Bloofield. USA. August.

Eco U. (1980), Segno, Arnoldo Mondadori Editore.

Eco U. (1979) Lector in fabula, Bompiani Ed.

Finkelstein A. \& Fuks H. 1989) "Multi-party Specification", Proceedings of the 5th Workshop on Software Specification and Design, Pittsburgh, IEEE C. S. Press.

Galarreta D., Charrel P.J., Rothenburger B \& al. (1998) "Study of Dynamic Viewpoints in Satellite Design", Proceedings of the $9^{\text {th }}$ Symposium IFAC Information COntrol in Manufacturing systems INCOM'98, Nancy, June 24-26.

Granger G.G. (1994), Formes, opérations, objets, VRIN Edirion. 
Greimas A.J. \& Courtes J. (1979) Sémiotique. Dictionnaire Raisonné de la théorie du Langage (tome 1), Hachette.

Greimas A.J. (1966) Sémantique structurale, Larousse, Paris.

Habermas J. (1991) Texte und contexte, Suhrkamp Verlag.

ITU Recommendation X.901-904 - ISO 10746 1-4, "Open Distributed Processing - Reference Model - Parts 14", July 1995.

Kontonya G. \&Sommerville I. (1992) "Viewpoints for Requirements Definition", IEEE Software Engineering Journal, Vol 7, pp. 375-387.

Leite J.C.S.P. \& Freeman P.A. (1991) "Requirements Validation Through Viewpoint Resolution", IEEE Transactions on Software Engineering, Vol 17(12).

Leite J.C.S.P. (1988) Viewpoint resolution in requirements elicitation, PhD Thesis, University of California, Irvine.

Lemoigne J.L. (1990) La modelisation des systèmes complexes, Dunod.

Levinas E. (1994) Nine Talmudic Readings, Indiana University Press.

Morin E. (1991) La complexité humaine, Champs l'Essentiel. Flammarion.

Mullery G.P. (1979) "CORE - a method for controlled requirement specifications", Proceedings of the $4^{\text {th }}$ International Conference on Software Engineering, München, Germany; IEEE CS Press, pp. 126-135.

Nuseibeh B., Kramer J. \& Finkelstein A. (1994) "Expressing the Relationships between Multiple Views in Requirements Specification", IEEE Transactions on Software Engineering, Vol 20(10), pp 760-773.

Peirce C.S. (1932) Collected papers, Harvard U.P.

Rohmer E., Chabrol C. (1988) Hitchcock the first forty-four films, Ungar film library.

Rumbaugh J., Jacobson I. \& Booch G. (1998) The Unified Modelling Language Reference Manual, AddisonWesley.

Zave P. (1997) "Classification of Research efforts in Requirement Engineering". Computing Surveys, Vol 29(4), pp.315-321. 